\title{
An inverse nodal problem for differential pencils with complex spectral parameter dependent boundary conditions
}

\author{
Tuba Gulsen, Emrah Yilmaz, Hikmet Koyunbakan \\ Department of Mathematics, Faculty of Science, Firat University, Elazig, Turkey \\ Received: 24 October 2016, Accepted: 19 December 2016 \\ Published online: 7 January 2017.
}

\begin{abstract}
In this study, we are concerned with an inverse nodal problem for second order differential pencil on a finite interval with complex spectral parameter dependent boundary conditions by using nodal points. We give some reconstruction formulas for potential functions $p$ and $q$ as a limit.
\end{abstract}

Keywords: Inverse nodal problem, differential pencil, Eigenvalues.

\section{Introduction}

Theory of inverse problems constitutes a vast field of mathematics. There are some approaches related to this subject; one of them is to study inverse eigenvalue problem (see [1], [2], [3], [4], [5], [6], [7]) and the other one is to study inverse nodal problem. The inverse nodal problem was introduced as a new type of spectral data which is so called nodal points-zeros of eigenfunctions by McLaughlin in 1988 [8]. She proved that the knowledge of a dense subset of nodal points of the eigenfunction can alone determine the potential function of Sturm-Liouville equation up to a constant. Independently, Shen studied the relations between the nodal points and the density function of string equation in 1988 [9]. Later, many authors have studied inverse nodal problem for different operators (see [10], [11], [12], [13], [14], [15], [16], [17], [18], [19], [20]).

In this study, we consider boundary value problem $L=L\left(p, q, h_{0}, h_{1}, H_{0}, H_{1}\right)$ of the form

$$
l_{\lambda} y(x, \lambda)=y^{\prime \prime}(x)+\left[\lambda^{2}-2 \lambda p(x)-q(x)\right] y(x)=0,0<x<\pi
$$

with the boundary conditions

$$
\begin{gathered}
y^{\prime}(0)+\left(i \lambda h_{1}+h_{0}\right) y(0)=0, \\
y^{\prime}(\pi)+\left(i \lambda H_{1}+H_{0}\right) y(\pi)=0,
\end{gathered}
$$

where $i^{2}=-1, \lambda$ is a spectral parameter; $p \in W_{1}^{2}[0, \pi], q \in L_{1}[0, \pi]$ are complex-valued functions; $h_{0}, h_{1}, H_{0}, H_{1} \in \mathbb{C}, h_{1} \neq \pm 1, H_{1} \neq \pm 1$ [21]. Equation (1) is also known as diffusion equation in literature.

Let us emphasize some historical and physical improvement of differential pencils. Jaulent and Jean [22] stated the actual background of diffusion operators and discussed the inverse problem for the diffusion equation. Also, Gasymov 
and Guseinov studied the spectral theory of this operator [23]. The problem of describing the interactions between colliding particles is of fundemental interesting in physics. In many cases, a description can be carried out through a well known theoretical model. In particular, one is interested in collisions of two spinless particles, and it is supposed that the $s$ - wave binding energies and $s$-wave scattering matrix are exactly known from collision experiments. $s-$ wave Schrödinger equation with a radial static potential $V$ can be written as

$$
y^{\prime \prime}+[E-V(x)] y=0, x \geq 0,
$$

where the potential function depends on energy in some way and has the following form of energy dependence

$$
V(x, E)=U(x)+2 \sqrt{E} Q(x) .
$$

$U(x)$ and $Q(x)$ are complex-valued functions. (3) reduces to the Klein-Gordon $s$-wave equation with the static potential $Q(x)$, for a particle of zero mass and the energy $\sqrt{E}$ with the additional condition $U(x)=-Q^{2}(x)$ [22]. The Klein Gordon equation is considered one of the most important mathematical models in quantum field theory. The equation appears in relativistic physics and is used to describe dispersive wave phenomena in general. The Klein-Gordon equation arise in physics in linear and nonlinear forms [24].

Differential equations with a nonlinear dependence on the spectral parameter frequently appear in mathematics as well as in applications. Some aspects of this type problems were studied by many authors (see [25], [26], [27], [28], [29], [30], [31], [32], [33], [34], [35], [36], [37], [38]). Such problems play an important role in mathematics and have many applications in natural sciences and engineering.

Let $\lambda_{n}$ be the $n$-th eigenvalue, $D_{0}^{\pi}$ be a circle with radius $\pi$, centered 0 and $x_{j}^{n}$ be the nodal points of the $n-$ th eigenfunction where $j=1,2, \ldots, n-1$ for the problem (1), (2). The numbers $x_{j}^{n}, j=1,2, \ldots, n-1$ are the nodal points of the eigenfunction $y\left(x, \lambda_{n}\right)$, then $y\left(x_{j}^{n}, \lambda_{n}\right)=0 . n-t h$ eigenfunction of the problem (1.1),(1.2) has exactly $n$-nodes inside the circle $D_{0}^{\pi}$. We assume that $l_{j}^{n}$ is the distance of two consecutive nodal points $x_{j}^{n}$ and $x_{j+1}^{n}, l_{j}^{n}=x_{j+1}^{n}-x_{j}^{n}$. Let us define the function $j_{n}(x)$ to be the largest index $j$ such that $0 \leq x_{j}^{n} \leq x$ for $n>0$, and the function $j_{n}(x)$ to be the largest index $j$ such that $0 \leq x \leq x_{j}^{n}$ for $n<0$. Thus, $j=j_{n}(x)$ iff $x \in\left[x_{j}^{n}, x_{j+1}^{n}\right)$ for $n>0$ and $x \in\left[x_{j+1}^{n}, x_{j}^{n}\right)$ for $n<0$ on $D_{0}^{\pi}$.

In this study, inverse nodal problem for differential pencil $L$ on a finite interval is studied. We have reconstructed the potential functions $p$ and $q$ from the nodal points of eigenfunctions as complex, provided $p, q$ are smooth enough.

\section{Main results}

In this section, we will try to obtain some asymptotic results for nodal parameters and some reconstruction formulas for potentials $p$ and $q$ which has been obtained as solution of an inverse nodal problem.

Lemma 1. [21] Let $\varphi(x, \lambda)$ and $\psi(x, \lambda)$ be the solutions to $l_{\lambda} y(x, \lambda)=0$ with the initial conditions

$$
\left(\varphi(0, \lambda), \varphi^{\prime}(0, \lambda)\right)=(0,1)=\left(\psi^{\prime}(0, \lambda), \psi(0, \lambda)\right)
$$

Then, the following representations hold:

$$
\begin{aligned}
\varphi(x, \lambda) & =\frac{\sin [\lambda x-\alpha(x)]}{\lambda}-b_{1}(x) \frac{\cos [\lambda x-\alpha(x)]}{\lambda^{2}}+a_{1}(x) \frac{\sin [\lambda x-\alpha(x)]}{\lambda^{2}} \\
& +b_{2}(x) \frac{\cos [\lambda x-\alpha(x)]}{\lambda^{3}}+a_{2}(x) \frac{\sin [\lambda x-\alpha(x)]}{\lambda^{3}}+o\left(\frac{e^{\tau x}}{\lambda^{3}}\right)
\end{aligned}
$$


and

$$
\begin{aligned}
\psi(x, \lambda) & =\cos [\lambda x-\alpha(x)]-c_{1}(x) \frac{\cos [\lambda x-\alpha(x)]}{\lambda}+b_{1}(x) \frac{\sin [\lambda x-\alpha(x)]}{\lambda} \\
& +d_{2}(x) \frac{\cos [\lambda x-\alpha(x)]}{\lambda^{2}}+d_{1}(x) \frac{\sin [\lambda x-\alpha(x)]}{\lambda^{2}}+o\left(\frac{e^{\tau x}}{\lambda^{2}}\right)
\end{aligned}
$$

where

$$
\begin{aligned}
& \alpha(x)=\int_{0}^{x} p(t) d t, b_{1}(x)=\frac{1}{2} \int_{0}^{x}\left[p^{2}(t)+q(t)\right] d t, a_{1}(x)=\frac{1}{2}[p(x)+p(0)], \\
& b_{2}(x)=\frac{1}{4}\left[p^{\prime}(x)-p^{\prime}(0)\right]-\frac{1}{2} b_{1}(x)[p(x)+p(0)]-\frac{1}{2} \int_{0}^{x} p(t)\left[p^{2}(t)+q(t)\right] d t, \\
& a_{2}(x)=\frac{1}{8}\left[5 p^{2}(x)+5 p^{2}(0)+2 p(0) p(x)\right]+\frac{q(x)+q(0)}{4}-\frac{1}{2} b_{1}^{2}(x), \\
& c_{1}(x)=\frac{1}{2}[p(0)-p(x)], \\
& d_{2}(x)=\frac{1}{8}\left[5 p^{2}(x)-2 p(0) p(x)-3 p^{2}(0)+2 q(x)-2 q(0)\right]-\frac{1}{2} b_{1}^{2}(x), \\
& d_{1}(x)=-\frac{1}{4}\left[p^{\prime}(x)+p^{\prime}(0)\right]+\frac{1}{2} b_{1}(x)[p(x)-p(0)]+\frac{1}{2} \int_{0}^{x} p(t)\left[p^{2}(t)+q(t)\right] d t,
\end{aligned}
$$

and $\tau=|\operatorname{Im} \lambda|$.

Lemma 2. [21] Let $\lambda_{n}, n \in A=\{ \pm 0, \pm 1, \pm 2, \ldots\}$, be the eigenvalues of the pencil $L$. Then, the sequence $\left\{\lambda_{n}: n \in A\right\}$ satisfies the following asymptotic expression as $|n| \rightarrow \infty$

$$
\lambda_{n}=n+\frac{1}{2 \pi i} \log \frac{\left(h_{1}+1\right)\left(H_{1}-1\right)}{\left(h_{1}-1\right)\left(H_{1}+1\right)}+\frac{k_{5}}{n+\frac{1}{2 \pi i} \log \frac{\left(h_{1}+1\right)\left(H_{1}-1\right)}{\left(h_{1}-1\right)\left(H_{1}+1\right)}}+\frac{k_{6}+k_{7}}{\left[n+\frac{1}{2 \pi i} \log \frac{\left(h_{1}+1\right)\left(H_{1}-1\right)}{\left(h_{1}-1\right)\left(H_{1}+1\right)}\right]^{2}}+O\left(\frac{1}{n^{3}}\right) .
$$

For the convenience, Yang sets

$$
\begin{aligned}
a_{1} & =a_{1}(\pi), b_{1}=b_{1}(\pi), c_{i}=c_{i}(\pi)(i=1,2,3), d_{i}=d_{i}(\pi)(i=1,2), e_{i}=e_{i}(\pi)(i=1,2) \\
k_{1} & =a_{1}+a_{1} h_{1} H_{1}+i\left(H_{1} b_{1}-h_{1} b_{1}-h_{0} H_{1}-h_{1} H_{0}\right), \\
k_{2} & =b_{1}-h_{0}+H_{0}-b_{1} h_{1} H_{1}-i c_{1}\left(h_{1}+H_{1}\right), \\
k_{3} & =e_{2}-\left(h_{0}-H_{0}\right) b_{1}+a_{2} h_{1} H_{1}-h_{0} H_{0}+i\left(H_{1} d_{1}-h_{1} c_{3}-a_{1} h_{0} H_{1}-a_{1} h_{1} H_{0}\right), \\
k_{4} & =e_{1}-h_{0} c_{1}-H_{0} c_{1}+b_{2} h_{1} H_{1}+i\left(H_{1} d_{2}-h_{1} c_{2}+b_{1} h_{0} H_{1}+b_{1} h_{1} H_{0}\right), \\
k_{5} & =-\frac{1}{\pi} \frac{i k_{1}\left(h_{1}-H_{1}\right)+k_{2}\left(h_{1} H_{1}-1\right)}{\left(h_{1} H_{1}-1\right)^{2}-\left(h_{1}-H_{1}\right)^{2}}, \\
k_{6} & =-\frac{1}{\pi} \frac{i k_{3}\left(h_{1}-H_{1}\right)+k_{4}\left(h_{1} H_{1}-1\right)}{\left(h_{1} H_{1}-1\right)^{2}-\left(h_{1}-H_{1}\right)^{2}}, \\
k_{7} & =\frac{\left[k_{1}\left(h_{1}-H_{1}\right) i+k_{2}\left(h_{1} H_{1}-1\right)\right]\left[k_{1}\left(h_{1}-H_{1}\right)-k_{2}\left(h_{1} H_{1}-1\right) i\right]}{\pi\left[\left(h_{1} H_{1}-1\right)^{2}-\left(h_{1}-H_{1}\right)^{2}\right]},
\end{aligned}
$$

where

$$
c_{2}(x)=-\frac{1}{8}\left[3 p^{2}(x)+2 p(0) p(x)-5 p^{2}(0)+2 q(x)-2 q(0)\right]-\frac{1}{2} b_{1}^{2}(x),
$$




$$
\begin{aligned}
& c_{3}(x)=-\frac{1}{4}\left[p^{\prime}(x)+p^{\prime}(0)\right]-\frac{1}{2} b_{1}(x)[p(x)-p(0)]+\frac{1}{2} \int_{0}^{x} p(t)\left[p^{2}(t)+q(t)\right] d t, \\
& e_{1}(x)=\frac{1}{4}\left[p^{\prime}(x)-p^{\prime}(0)\right]-\frac{1}{2} b_{1}(x)[p(x)+p(0)]+\frac{1}{2} \int_{0}^{x} p(t)\left[p^{2}(t)+q(t)\right] d t
\end{aligned}
$$

and

$$
e_{2}(x)=\frac{1}{8}\left[3 p^{2}(x)-2 p(0) p(x)+3 p^{2}(0)+2 q(x)+2 q(0)\right]+\frac{1}{2} b_{1}^{2}(x) .
$$

Theorem 1. Suppose that $p \in W_{1}^{2}[0, \pi]$ and $q \in L_{1}[0, \pi]$, then

$$
x_{j}^{n}=\frac{\pi j}{n}+\frac{j \log A}{2 n^{2} i}+\frac{1}{n} \int_{0}^{x_{j}^{n}} p(t) d t+\frac{\log A}{2 n^{2} i \pi} \int_{0}^{x_{j}^{n}} p(t) d t+\frac{1}{2 n^{2}} \int_{0}^{x_{j}^{n}}\left[p^{2}(t)+q(t)\right] d t+O\left(\frac{1}{n^{3}}\right),
$$

and

$$
l_{j}^{n}=\frac{\pi}{n}+\frac{\log A}{2 n^{2} i}+\frac{1}{n} \int_{x_{j}^{n}}^{x_{j+1}^{n}} p(t) d t+\frac{\log A}{2 n^{2} i \pi} \int_{x_{j}^{n}}^{x_{j+1}^{n}} p(t) d t+\frac{1}{2 n^{2}} \int_{x_{j}^{n}}^{x_{j+1}^{n}}\left[p^{2}(t)+q(t)\right] d t+O\left(\frac{1}{n^{3}}\right),
$$

where $A=\frac{\left(h_{1}+1\right)\left(H_{1}-1\right)}{\left(h_{1}-1\right)\left(H_{1}+1\right)}$ and $i^{2}=-1$.

Proof. We will prove only for $\varphi(x, \lambda)$. The other case for $\psi(x, \lambda)$ can be proved similarly. If $\varphi(x, \lambda)$ equals to zero, then as long as $\cos [\lambda x-\alpha(x)]$ is not close to 0 ,

$$
0=\frac{\tan [\lambda x-\alpha(x)]}{\lambda}-\frac{b_{1}(x)}{\lambda^{2}}+a_{1}(x) \frac{\tan [\lambda x-\alpha(x)]}{\lambda^{2}}+\frac{b_{2}(x)}{\lambda^{3}}+a_{2}(x) \frac{\tan [\lambda x-\alpha(x)]}{\lambda^{3}}+O\left(\frac{1}{\lambda^{3}}\right) .
$$

Hence

$$
\tan [\lambda x-\alpha(x)]\left[1+O\left(\frac{1}{\lambda}\right)\right]=\frac{b_{1}(x)}{\lambda}-\frac{b_{2}(x)}{\lambda^{2}}+O\left(\frac{1}{\lambda^{2}}\right) .
$$

Now take $\lambda=\lambda_{n}$ and $x=x_{j}^{n}$. Hence by Taylor's expansion for the tangent function, we get

$$
\lambda_{n} x_{j}^{n}-\alpha(x)=\pi j+\frac{b_{1}(x)}{\lambda_{n}}-\frac{b_{2}(x)}{\lambda_{n}^{2}}+O\left(\frac{1}{\lambda_{n}^{2}}\right)
$$

or

$$
x_{j}^{n}=\frac{\alpha(x)}{\lambda_{n}}+\frac{\pi j}{\lambda_{n}}+\frac{b_{1}(x)}{\lambda_{n}^{2}}-\frac{b_{2}(x)}{\lambda_{n}^{3}}+O\left(\frac{1}{\lambda_{n}^{3}}\right) .
$$

And, by using estimates of $b_{1}(x), b_{2}(x)$ and $\alpha(x)$, it yields

$$
x_{j}^{n}=\frac{\pi j}{\lambda_{n}}+\frac{1}{\lambda_{n}} \int_{0}^{x_{j}^{n}} p(t) d t+\frac{1}{2 \lambda_{n}^{2}} \int_{0}^{x_{j}^{n}}\left[p^{2}(t)+q(t)\right] d t-\frac{b_{2}\left(x_{j}^{n}\right)}{\lambda_{n}^{3}}+O\left(\frac{1}{\lambda_{n}^{3}}\right) .
$$

Furthermore, by using following asymptotic formulas in (7)

$$
\begin{aligned}
& \frac{1}{\lambda_{n}}=\frac{1}{n}+\frac{\log A}{2 n^{2} \pi i}+\frac{k_{5}}{n^{2}\left(n+\frac{1}{2 \pi i} \log A\right)}+O\left(\frac{1}{n^{4}}\right) \\
& \frac{1}{\lambda_{n}^{2}}=\frac{1}{n^{2}}-\frac{(\log A)^{2}}{4 n^{4} \pi^{2}}+\frac{k_{5}^{2}}{n^{4}\left(n+\frac{1}{2 \pi i} \log A\right)}+\frac{\log A}{n^{3} \pi i}+\frac{2 k_{5}}{n^{3}\left(n+\frac{1}{2 \pi i} \log A\right)}+\frac{(\log A) k_{5}}{n^{4} \pi i\left(n+\frac{1}{2 \pi i} \log A\right)}+O\left(\frac{1}{n^{5}}\right)
\end{aligned}
$$


and

$$
\frac{1}{\lambda_{n}^{3}}=\frac{1}{n^{3}}-\frac{3(\log A)^{2}}{4 n^{5} \pi^{2}}+\frac{\log A}{n^{4} \pi i}+\frac{2 k_{5}}{n^{4}\left(n+\frac{1}{2 \pi i} \log A\right)}+\frac{\log A}{2 n^{4} \pi i}+\frac{k_{5}}{n^{4}\left(n+\frac{1}{2 \pi i} \log A\right)}+O\left(\frac{1}{n^{6}}\right)
$$

we conclude that

$$
x_{j}^{n}=\frac{\pi j}{n}+\frac{j \log A}{2 n^{2} i}+\frac{1}{n} \int_{0}^{x_{j}^{n}} p(t) d t+\frac{\log A}{2 n^{2} i \pi} \int_{0}^{x_{j}^{n}} p(t) d t+\frac{1}{2 n^{2}} \int_{0}^{x_{j}^{n}}\left[p^{2}(t)+q(t)\right] d t+O\left(\frac{1}{n^{3}}\right) .
$$

Equality (5) gives the asymptotic expansion for nodal lengths $l_{j}^{n}$ as

$$
l_{j}^{n}=\frac{\pi}{n}+\frac{\log A}{2 n^{2} i}+\frac{1}{n} \int_{x_{j}^{n}}^{x_{j+1}^{n}} p(t) d t+\frac{\log A}{2 n^{2} i \pi} \int_{x_{j}^{n}}^{x_{j+1}^{n}} p(t) d t+\frac{1}{2 n^{2}} \int_{x_{j}^{n}}^{x_{j+1}^{n}}\left[p^{2}(t)+q(t)\right] d t+O\left(\frac{1}{n^{3}}\right) .
$$

Lemma 3. [12] Suppose that $q \in L_{1}[0, \pi]$. Then, for almost every $x \in[0, \pi]$ with $j=j_{n}(x)$

$$
\lim _{n \rightarrow \infty} \lambda_{n} \int_{x_{j}^{n}}^{x_{j+1}^{n}} q(t) d t=q(x)
$$

Theorem 2. Let $q \in L_{1}[0, \pi]$, then

$$
q(x)=\lim _{n \rightarrow \infty}\left[2 n^{2}\left(n l_{j}^{n}-\pi\right)-\frac{n}{i} \log A-p(x)\left(2 n+\frac{1}{\pi i}\right)-p^{2}(x)\right]
$$

where

$$
p(x)=\lim _{n \rightarrow \infty} n\left(n l_{j}^{n}-\frac{\log A}{2 n i}-\pi\right)
$$

and $i^{2}=-1$.

Proof. Considering (6) in the form

$$
l_{j}^{n}=\frac{\pi}{n}+\frac{\log A}{2 n^{2} i}+\frac{1}{n} \int_{x_{j}^{n}}^{x_{j+1}^{n}} p(t) d t+O\left(\frac{1}{n^{2}}\right)
$$

so that

$$
n \pi\left(\frac{n}{\pi} l_{j}^{n}-\frac{\log A}{2 n \pi i}-1\right)=n \int_{x_{j}^{n}}^{x_{j+1}^{n}} p(t) d t+O\left(\frac{1}{n}\right) .
$$

By Lemma 3 , for almost every $x \in[0, \pi]$, we obtain

$$
p(x)=\lim _{n \rightarrow \infty} n\left(n l_{j}^{n}-\frac{\log A}{2 n i}-\pi\right) .
$$


Now, we will get a reconstruction formula for the potential function $q$. Let consider (6) in the form

$$
l_{j}^{n}=\frac{\pi}{n}+\frac{\log A}{2 n^{2} i}+\frac{1}{n} \int_{x_{j}^{n}}^{x_{j+1}^{n}} p(t) d t+\frac{\log A}{2 n^{2} i \pi} \int_{x_{j}^{n}}^{x_{j+1}^{n}} p(t) d t+\frac{1}{2 n^{2}} \int_{x_{j}^{n}}^{x_{j+1}^{n}}\left[p^{2}(t)+q(t)\right] d t+O\left(\frac{1}{n^{3}}\right) .
$$

After some algebraic computations, we can get

$$
n \pi\left(\frac{n}{\pi} l_{j}^{n}-1\right)=\frac{\log A}{2 i}+n \int_{x_{j}^{n}}^{x_{j+1}^{n}} p(t) d t+\frac{\log A}{2 i \pi} \int_{x_{j}^{n}}^{x_{j+1}^{n}} p(t) d t+\frac{1}{2} \int_{x_{j}^{n}}^{x_{j+1}^{n}}\left[p^{2}(t)+q(t)\right] d t+O\left(\frac{1}{n}\right),
$$

and

$$
2 n^{2}\left(n l_{j}^{n}-\pi\right)=\frac{n}{i} \log A+2 n p(x)+\frac{p(x)}{\pi i}+n \int_{x_{j}^{n}}^{x_{j+1}^{n}}\left[p^{2}(t)+q(t)\right] d t+O(1),
$$

for sufficiently large $n$. Hence, by using Lemma 3, it yields

$$
q(x)=\lim _{n \rightarrow \infty}\left[2 n^{2}\left(n l_{j}^{n}-\pi\right)-\frac{n}{i} \log A-p(x)\left(2 n+\frac{1}{\pi i}\right)-p^{2}(x)\right] .
$$

This completes the proof.

\section{Conclusion}

In this study, we give some results about inverse nodal problem for differential pencils with complex spectral parameter in boundary conditions. Asymptotic formulas of nodal parameters and potential functions for this problem are obtained. We think that it will offer a different perspective to spectral theory.

\section{Competing interests}

The authors declare that they have no competing interests.

\section{Authors' contributions}

All authors have contributed to all parts of the article. All authors read and approved the final manuscript.

\section{References}

[1] V. A. Ambartsumyan, Über eine Frage der Eigenwerttheorie, Zeitschrift für Physik, (1929) 53, 690-695.

[2] B. M. Levitan and I. S. Sargsjan, Introduction to Spectral Theory: Self Adjoint Ordinary Differential Operators, American Mathematical Society, Providence, Rhode Island, (1975).

[3] V. Pivovarchik, Direct and inverse three-point Sturm-Liouville problems with parameter dependent boundary conditions, Asymptotic Analysis, (2001) 26(3-4), 219-238.

[4] C. T. Shieh, S. A. Buterin and M. Ignatiev, On Hochstadt-Lieberman theorem for Sturm-Liouville operators, Far East Journal of Applied Mathematics, (2011) 52(2), 131-146. 
[5] R. Hryniv and N. Pronska, Inverse spectral problems for energy-dependent Sturm-Liouville equations, Inverse Problems, (2012) 28(8), 085008 .

[6] G. Freiling and V. A. Yurko, Inverse Sturm-Liouville problems and their applications, NOVA Science Publishers, New York, (2001).

[7] J. Pöschel and E. Trubowitz, Inverse spectral theory, volume 130 of Pure and Applied Mathematics, Academic Press, Inc, Boston, MA, (1987).

[8] J. R. McLaughlin, Inverse spectral theory using nodal points as data-a uniqueness result, Journal of Differential Equations, (1988) 73(2), 354-362.

[9] C. L. Shen, On the nodal sets of the eigenfunctions of the string equation, SIAM Journal on Mathematical Analysis, (1988) 19(6), 1419-1424.

[10] C. K. Law and J. Tsay, On the well-posedness of the inverse nodal problem, Inverse Problems, (2001) 17(5), 1493-1512.

[11] Y. H. Cheng and C. K. Law, The inverse nodal problem for Hill's Equation, Inverse Problems, (2006) 22(3), 891-901.

[12] C. K. Law and C. F. Yang, Reconstructing of the potential function and its derivatives using nodal data, Inverse Problems, (1999) 14(2), 299-312.

[13] O. H. Hald and J. R. McLaughlin, Solutions of inverse nodal problems, Inverse Problems, (1989) 5(3), 307-347.

[14] W. C. Wang, Y. H. Cheng and W. C. Lian, Inverse nodal problems for the $p$-Laplacian with eigenparameter dependent boundary conditions, Mathematical and Computer modelling, (2011) 54(11-12), 2718-2724.

[15] C. T. Shieh and V. A. Yurko, Inverse nodal and inverse spectral problems for discontinuous boundary value problems, Journal of Mathematical Analysis and Applications, (2008) 347(1), 266-272.

[16] C. F. Yang, Inverse nodal problems for the Sturm-Liouville operator with eigenparameter dependent boundary conditions, Operators and Matrices, (2012) 6(1), 63-77.

[17] H. Koyunbakan, E. S. Panakhov, A uniqueness theorem for inverse nodal problem, Inverse Problems in Science and Engineering, (2007) 15(6), 517-524.

[18] S. A. Buterin and C. T. Shieh, Inverse nodal problem for differential pencils, Applied Mathematics Letters, (2009) 22(8), 12401247.

[19] E. Yilmaz and H. Koyunbakan, Reconstruction of potential function and its derivatives for Sturm-Liouville problem with eigenvalues in boundary condition, Inverse Problems in Science and Engineering, (2010) 18(7), 935-944.

[20] Y. V. Kuryshova and C. T. Shieh, An inverse nodal problem for integro-differential operators, Journal of Inverse and III-posed Problems, (2010) 18(4), 357-369.

[21] C. F. Yang, Trace Formulae for differential pencils with spectral parameter dependent boundary conditions, Mathematical Methods in the Applications, (2013) 37(9), 1325-1332.

[22] M. Jaulent and C. Jean, The inverse s-wave scattering problem for a class of potentials depending on energy, Communications in Mathematical Physics, (1972) 28(3), 177-220.

[23] M. G. Gasymov and G. Sh. Guseinov, Determination of a diffusion operator from the spectral data, Doklady Akademii Nauk Azerbaijan SSR, (1981) 37(2), 19-23.

[24] A. M. Wazwaz, Partial differential equations: Methods and applications, Balkema, The Netherlands, (2002).

[25] L. K. Sharma, P. V. Luhanga and S. Chimidza, Potentials for the Klein-Gordon and Dirac equations, Chiang Mai Journal of Science, (2011) 38(4), 514-526.

[26] K. Chadan, D. Colton, L. Päivärinta and W. Rundell, An introduction to inverse scattering and inverse spectral problems, Dokl. Akad. Nauk SSSR, (1985), 285(6), 1292-1296; Society for Industrial and Applied Mathematics, Philadelphia, PA, (1997).

[27] G. Sh. Guseinov, On spectral analysis of a quadratic pencil of Sturm-Liouville operators, Soviet Mathematics Doklady, (1985) 32(3), 859-862.

[28] I. M. Guseinov and I. M. Nabiev, A class of inverse problems for a quadratic pencil of Sturm-Liouville operators, Differential Equations, (2000) 36(3), 471-473.

[29] I. M. Guseinov and I. M. Nabiev, The inverse spectral problem for pencils of differential operators, Sbornik Mathematics, (2007) 198(11), 1579-1598.

[30] F. G. Maksudov and G. Sh. Guseinov, On solution of the inverse scattering problem for a quadratic pencil of one dimensional Schrödinger operators on thewhole axis, Soviet Mathematics Doklady, (1987) 34, 34-38.

[31] I. M. Nabiev, Multiplicities and relation position of eigenvalues of a quadratic pencil of Sturm-Liouville operators, Mathematical Notes, (2000) 67(3), 309-319. 
[32] C. F. Yang and A. Zettl, Half inverse problems for quadratic pencils of Sturm-Liouville operators, Taiwanese Journal of Mathematics, (2012) 16(5), 1829-1846.

[33] E. Bairamov, Ö. Çakar and A. O. Çelebi, Quadratic pencil of Schrödinger operators with spectral singularities: discrete spectrum and principal functions, Journal of Mathematical Analysis and Applications, (1997) 216(1), 303-320.

[34] H. Koyunbakan, Reconstruction of potential function for diffusion operator, Numerical Functional Analysis and Optimization, (2009) 30(1-2), 111-120.

[35] H. Koyunbakan and E. Yilmaz, Reconstruction of the potential function and its derivatives for the diffusion operator, Zeitschrift für Naturforchung A, (2008) 63(3-4), 127-130.

[36] C. F. Yang, Reconstruction of the diffusion operator from nodal data, Zeitschrift für Naturforchung A, (2010) 65(1-2), 100-106.

[37] R. Kh. Amirov and A. A. Nabiev, Inverse Problems for the Quadratic Pencil of the Sturm-Liouville Equations with Impulse, Abstract and Applied Analysis, Volume 2013, Article ID 361989, 10 pp.

[38] Y. P. Wang, The inverse problem for differential pencils with eigenparameter dependent boundary conditions from interior spectral data, Applied Mathematics Letters, (2012) 25(7), 1061-1067. 